\title{
Illusions and aftereffects of length in the minimal form of the parallel-lines configuration: A reexamination
}

\author{
JOHN PREDEBON \\ University of Sydney, Sydney, New South Wales, Australia
}

\begin{abstract}
A number of recent studies have used the graded-series method to measure the illusion and aftereffect distortions of perceived length in the minimal form of the parallel-lines configuration. However, because of some peculiar characteristics of the graded-series method, the aftereffect distortions measured with this method cannot be interpreted unequivocally as reflecting distortions of the perceived length of the test line. On the basis of the results of an experiment that examined the contribution of a possible confound present in the method, it is argued that the method should not be used to measure length aftereffects, and, furthermore, that the results of studies using this method need to be reconsidered.
\end{abstract}

Recent studies (Brigell \& Uhlarik, 1979; Jordan \& Haleblian, 1988; Jordan \& Uhlarik, 1985) have used the minimal form of the parallel-lines illusion configuration shown in Figure 1 to compare the distortions of perceived length produced either by the simultaneous presentation of a single contextual line (illusion paradigm) or by the prior inspection of a single adapting line (aftereffect paradigm). The results of these studies indicate that the aftereffect and illusion occur in opposite directions. In the illusion paradigm, the test line's length is underestimated in the presence of a shorter contextual line, and overestimated in the presence of a longer contextual line. Conversely, in the aftereffect paradigm the test line is underestimated if it is shorter, and overestimated if it is longer, than a previously inspected adapting line. Descriptively, these distortions may be summarized as assimilation effects in the illusion paradigm (i.e., the perceived length of the test line is shifted toward the length of the contextual line) and contrast effects in the aftereffect paradigm (i.e., the perceived length of the test line is shifted away from the adapting line).

The studies cited above have used the graded-series comparison scale method to measure distortions of length. This method requires observers to select, from a graded set of simultaneously presented comparison lines, the line that appears equal in length to the test line. The lengths of the comparison lines span the length of the test line, with the middle line physically matching the length of the test line. In these studies, the experimenter varies across trials the length of the adapting line (aftereffect paradigm) or the length of the contextual line (illusion paradigm) while keeping constant the lengths of the test and comparison lines. The comparison lines are oriented horizontally and positioned to one side of the horizontally

Address correspondence to John Predebon, Department of Psychology, University of Sydney, Sydney, NSW, 2006, Australia.

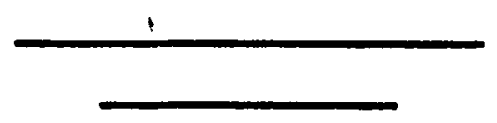

Figure 1. Minimal form of the parallel-lines illusion. The shorter bottom line produces underestimation of the top line's length, and, similarly, the longer top line results in an overestimation of the bottom line's length.

presented test line; in the illusion paradigm the comparison line array is presented simultaneously with the parallel-lines figure, and in the aftereffect paradigm it is presented simultaneously with the single test line. The data for analysis are the observers' judgments converted to deviations from the point of subjective equality (PSE) for a "no-context" or control line of the same length as the test line. The PSE, determined prior to the experimental session, provides a baseline for evaluating the effects of the experimental manipulations. In this communication I raise some observations regarding the appropriateness of using the graded-series scale method to measure distortions of perceived length. I also report an experiment in which I attempted to validate the results obtained with the particular version of the graded-series scale method used in the above studies.

In its application to measuring the magnitude of length illusions, the graded-series method seems unproblematical. There is, however, a minor complication; the comparison scale itself is illusion inducing, as evidenced by the consistent finding that, in the absence of either prior (aftereffect) or simultaneous (illusion) exposure to a test line, a single line is matched to a physically shorter line in the comparison line array. Although it has been decribed (e.g., Brigell \& Uhlarik, 1979) as a perceptual underestimation of the test line, this finding can also be decribed as a perceptual overestimation of the comparison lines, perhaps as a consequence of simultaneous assimilatory interactions between the comparison lines. This dis- 
tortion within the scale (hereafter also referred to as the scale illusion) is unlikely to confound the measurement of illusion magnitude, for three reasons. First, there is no plausible reason for expecting the magnitude of the scale illusion to be affected by the illusion being measured; in this sense the measurement device is independent of the phenomenon under investigation. Second, as used in the above studies, the scale illusion is factored out by the procedure of converting judgments of the length of the test line to deviations from the PSE. Third, the gradedseries method has been shown to produce results similar to those of other methods for measuring illusion magnitude (Coren \& Girgus, 1972).

The situation with respect to measuring aftereffect distortions is far less straightforward; indeed, it is not at all obvious whether this method, at least in the manner used in the above studies, should be expected to detect any aftereffect-induced distortions whatsoever. In the aftereffect paradigm, observers adapt to a single line by fixating for a period of time on a point positioned, say, slightly below the line. This display is then replaced by the test line and the comparison line array, and the observer matches the length of the test line to a line in the comparison display. The interesting and distinctly odd feature of this matching procedure is that the observer is allowed to inspect the comparison array and the test line with free regard; that is, unlike the adapting phase, there is no restriction on observers' eye-movements during the matching phase. The oddity of this procedure is best highlighted by considering the paradigmatic example of an observer adjusting the length of a single comparison line to match the length of the test line. According to models of length aftereffects, including cognitive-judgmental (e.g., Coren \& Girgus, 1978) and physiological (e.g., Brigell \& Uhlarik, 1979) models, the aftereffect distortion is contour nonspecific in the sense that it affects the perceived length of any visually glanced contour of similar orientation to the adapting line whose image falls on or near the previously adapted retinal area. In this example, for the two lines to appear equal in length, the comparison line must be adjusted to physical equality with the test line; in scanning the comparison and test lines, both lines will be subject to the aftereffect distortion, in which case no distortion, whether it be assimilation or contrast, can be detected by this method. Indeed, any departure from the point of physical equality would raise issues of experimental artifacts, response biases, and so forth. Obviously, these considerations apply to any method for measuring aftereffects that allows free inspection of the test and comparison lines. From this perspective there does not appear to be any theoretically tenable reason for expecting the graded-series method to detect aftereffect-induced distortions of length. How, then, can the systematic pattern of aftereffect distortions found in the above studies be explained? One pragmatic response is to assert that the method works; for whatever reasons, the aftereffect distortion affects the perceived length of a subsequently presented single test line but not the perceived lengths of the comparison lines. Such a response, however, is both unenlightening and a mere restatement of the data collected with this method.

If the systematic pattern of errors found with the gradedseries method does index some kind of perceptual distortion in the aftereffect display, is it possible to retain the assumption that the aftereffect distortion equally affects the perceived lengths of the test line and comparison lines? The preceding analysis has omitted considering the possible effect of the illusory distortion within the scale itself, that is, the inherent perceptual lengthening of the comparison lines. As noted earlier, the scale illusion is unlikely to contaminate measurements of illusion magnitude. In the aftereffect paradigm, however, it is plausible to expect some summation of the scale-illusion and aftereffect distortions. In the relevant studies, the adapting line is longer or shorter than either the test line or the comparison lines. Hence, an aftereffect that produces perceptual lengthening of the test line will also perceptually lengthen the comparison lines; in the latter case the aftereffect distortion is expected to increase the magnitude of the distortion already present in the comparison line array. Conversely, an aftereffect that produces perceptual shortening of the test line will counteract, if not reverse the direction of, the scale illusion. Thus, unlike the illusion paradigm, in the aftereffect paradigm the magnitude of the distortion within the measurement device is not independent of the effect being measured. Consequently, although the aftereffect data obtained with the graded-series method may index perceptual distortions, it is impossible to determine whether the pattern of errors reflects distortions in the perceived length of the test line, distortions within the scale, or both.

In the present study the aftereffect form of the parallellines illusion was used to examine whether the contrast effects found with the graded-series method are also obtained with a slightly modified method that excludes, or at least minimizes, interactions between the aftereffect and the comparison scale. This was done by comparing the results obtained with the standard and modified versions of the graded-series method. In the standard, or identically-oriented-lines, method, both the test/adapting lines and comparison lines are oriented horizontally; in the modified, or orthogonally-oriented-lines, method, the test/adapting lines and comparison lines are oriented horizontally and vertically, respectively. If, as seems reasonable to suppose, length aftereffects are orientation dependent-that is, distortions of perceived length occur only for test lines oriented similarly to the adapting linethen adapting to a horizontal line should affect the perceived length of the horizontal test line but not the lengths of the vertically oriented comparison lines. Thus, with the modified method, interactions between the measurement scale and the phenomenon of interest are minimized. The standard method was expected to replicate the usual length contrast effect, that is, under- and overestimation of the test line for longer and shorter adapting lines, respectively. Of primary interest, however, was whether the modified method yields a pattern of errors similar to those measured by the standard method, as should be the 
case if the standard method does not involve interactions between the aftereffect distortion and the comparison scale. Additionally, in order to provide a basis for comparison, the study also measured, with both the standard and modified graded-series methods, the length distortions in the parallel-lines illusion.

\section{METHOD}

\section{Subjects}

Forty undergraduate psychology students participated in partial fullfilment of course requirements.

\section{Stimulus and Materials}

The stimulus lines were oriented horizontally and were presented on the screen of a Macintosh Plus computer. The length of the test line was $3 \mathrm{~cm}$ and the lengths of the four contextual/adapting lines were $1.5,2.0,4.5$, and $6.0 \mathrm{~cm}$ (1 mm equals approximately 3 pixels). All lines were $1 \mathrm{~mm}$ wide. In the illusion condition, the test and contextual lines were presented simultaneously. The test line was centered on the Macintosh screen and the contextual line was centered $1 \mathrm{~cm}$ below the test line. In the aftereffect condition, the test line was preceded by a single adapting line, located $1 \mathrm{~cm}$ above a small fixation point centered on the screen.

The two comparison scales each contained 15 black parallel lines, labeled A-O, which ranged in length from 2.3 to $3.7 \mathrm{~cm}$ in $1-\mathrm{mm}$ increments. The lines were $1 \mathrm{~mm}$ thick and were separated by $5 \mathrm{~mm}$. The $3-\mathrm{cm}$ middle line of the scale corresponded to the actual length of the test line. The two scales differed in the orientation of the comparison lines, either vertical or horizontal; the length of the lines increased from top to bottom for the horizontal scale and from left to right for the vertical scale. Separate transparencies of these scales could be attached to the milk-glass screen side of an otherwise light-tight box. This box was to the right of the Macintosh screen and was positioned so that the left end of the horizontal comparison-line array and the left line of the vertical comparison array were located $20 \mathrm{~cm}$ from the midpoint of the test line on the computer screen.

The subjects viewed the computer screen and comparison box through two rectangular cutouts $(17 \mathrm{~cm}$ wide and $10 \mathrm{~cm}$ high) in a black cardboard screen ( $60 \mathrm{~cm}$ and $50 \mathrm{~cm}$ high), which was positioned directly in front of the computer and comparison box. The seated subject, with head resting in a chin- and forehead rest, viewed the stimuli from a distance of $1 \mathrm{~m}$. Apart from the light of the computer screen and the comparison box, the experiment was conducted under darkened conditions. The luminance of the bright areas of both the Macintosh and comparison screens was $3.6 \mathrm{~cd} / \mathrm{m}^{2}$.

There were 6 practice trials designed to familiarize the subjects with using the graded-series scale and to determine the PSE for the test line (see Results). Each practice trial contained a single line, the length of which was $2.5,2.7,3.0,3.0,3.3$, or $3.5 \mathrm{~cm}$. To encourage the subjects to use a wide range of the response scale, the test session included, in addition to the $3-\mathrm{cm}$ test line, two filler lines. The lengths of these lines were 3.4 and $2.6 \mathrm{~cm}$, and they were paired randomly with either a longer or shorter adapting/contextual line. Judgments of these filler items were not included in the data analysis.

\section{Procedure and Design}

All subjects participated in two sessions, separated by an interval of at least 1 week. They were allocated randomly to either the illusion or the aftereffect paradigm. The standard graded-series method was used in the first session and the modified method in the second session.

Session 1. The subjects were acquainted with the experimental setup and procedure. The task was to match the apparent length of the test line to one of the lines in the comparison series by nam- ing the appropriate letter. In the aftereffect condition the test line was a single line, and in the illusion condition it was the upper line of the parallel-lines configuration. All subjects completed the six practice trials first, followed by the test trials.

The sequencing of the test trials in the illusion condition was as follows. The word READY appeared on the screen for $2 \mathrm{sec}$, after which it was replaced by the parallel-lines illusion configuration, that is, the test/filler and contextual lines, for up to $10 \mathrm{sec}$. The subject was required to make a judgment during this period. After an interval of $10 \mathrm{sec}$ the word READY appeared on the screen for the next trial. There were three random-order presentations of the four test and two filler stimuli, with an interval of $2 \mathrm{~min}$ between random orders. The aftereffect condition was similar to the illusion condition. There were three random-order presentations of the test and filler stimuli, with a 2 -min interval between random orders. The commencement of a trial was signaled by the appearance of the word FIXATION on the screen for $2 \mathrm{sec}$, followed by the adapting line for $60 \mathrm{sec}$, during which time the subject fixated the small mark in the center of the screen. This was then replaced by the test/filler line; as in the illusion condition, by freely inspecting the test line and comparison display, the subjects were required to make a length judgment within $10 \mathrm{sec}$. There was an intertrial break of $20 \mathrm{sec}$, after which the word READY reappeared on the screen to signal the next trial.

Subjects in both the illusion and aftereffect conditions made a total of 24 judgments: 6 practice judgments and 3 judgments of each of the four test and two filler stimuli.

Session 2. Apart from the vertically oriented comparison scale, the procedure was identical to that of Session 1 .

\section{RESULTS}

The average of the two length judgments of the 3-cm no-context line presented during the practice trials provided a measure of each subject's PSE for the 3-cm test line. For the first session the means of these judgments were 28.83 and $28.57 \mathrm{~mm}$ for the illusion and aftereffect conditions, respectively. This underestimation of the length of the no-context line confirms the results of earlier studies, although the magnitude of the matching error is somewhat smaller in the present study. The second-session means were 27.32 and $26.94 \mathrm{~mm}$ for the aftereffect and illusion conditions, respectively. The greater underestimation of the no-context line in the second session presurnably reflects a component due to the horizontal-vertical illusion.

For each session, each subject's judgments of the test line's length were averaged across the three presentations of the test stimulus, and these mean judgments were converted to deviations from PSE by subtracting the subject's PSE for that session from the mean judged length of each test stimulus. Thus, there were eight deviation scores for each subject, four for each session. The scores of each session and condition were averaged across the 20 subjects, and these data are presented in Figure 2.

The results of the standard procedure conditions (Session 1) are shown in Figure $2 A$, and they confirm earlier findings (e.g., Jordan \& Uhlarik, 1985). Assimilation of test-line length is evident in the illusion condition; contextual lines shorter than the test line resulted in overestimation of test length, and lines longer than the test line tended to produce underestimation. The aftereffect condition produced contrast of test length; adapting lines 


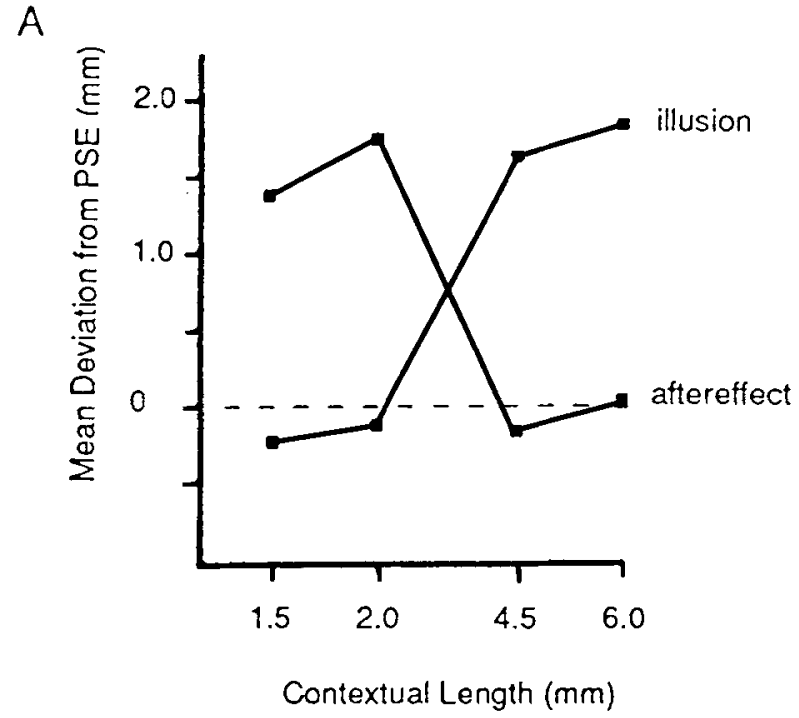

B

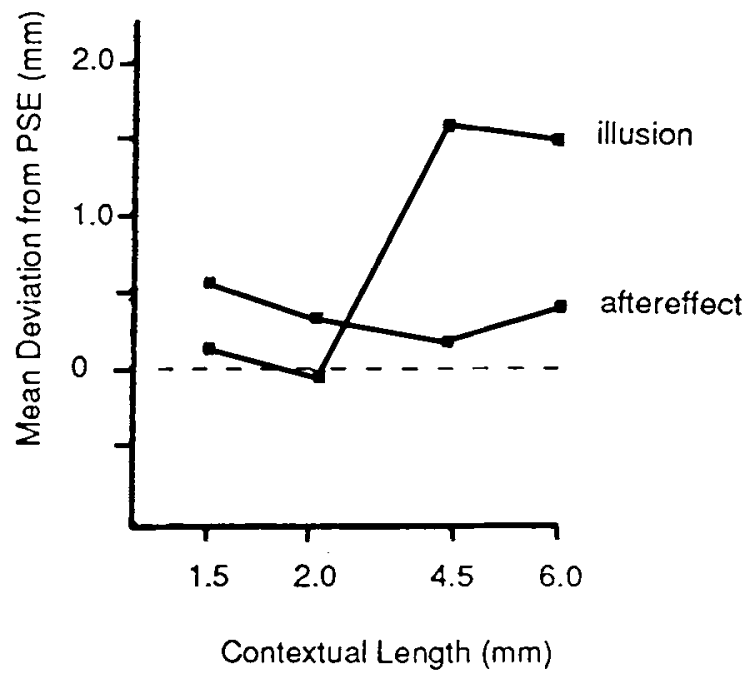

Figure 2. Mean deviations from point of subjective equality (PSE), in millimeters, for the illusion and aftereffect conditions. $A$ and $B$ show the data obtained with the standard and the modified gradedseries methods, respectively.

shorter than the test line resulted in overestimation of the test line, and adapting lines longer than the test line resulted in underestimation. The one-way repeated measures analysis of variance carried out separately on the illusion and the aftereffect data confirm these observations. This analysis was significant on both the illusion and aftereffect data $[F(3,57)=26.11$ and $27.56, p<.05$, respectively $]$, indicating that in both conditions test judgments were influenced by contextual/adapting line length.

Figure 2B shows the data obtained with the modified procedure (Session 2). The one-way repeated measures analyses of variance were significant on the illusion data $[F(3,57)=12.68, p<.05]$, but not on the aftereffect data $[F(3,57)=.63, p>.05]$, indicating that variations of contextual/adapting length differentially influenced test judgments in the illusion but not in the aftereffect condition. In the illusion condition, the pattern of errors describes length assimilation, whereas in the aftereffect condition neither length contrast nor length assimilation describes the data. ${ }^{1}$

\section{DISCUSSION}

The results of the present study can be summarized as follows: the illusion paradigm produces assimilation of test length with both graded-series methods, and the aftereffect paradigm yields length contrast with the standard method and neither length contrast nor length assimilation with the modified method. Taken together, these results indicate that the relative orientation of the comparison line and test/adapting lines influences the pattern of errors in the aftereffect, but not in the illusion paradigm.

The main purpose of this study was to evaluate a possible confound present in the standard graded-series method for measuring length distortions, in which the adapting line and comparison lines are oriented identically. In the aftereffect paradigm this affords an interaction between the aftereffect distortion and the scale illusion within the comparison scale, in which case measurement of the perceived length of the test line is confounded with the measurement device itself. The modified method overcomes this potential confound by presenting the test line and comparison lines at orthogonal orientations. The major finding of significance is that the modified method yields a pattern of aftereffect distortions quite unlike the pattern obtained with the standard method, thereby giving credence to the claim that the standard method does not provide a pure measure of the perceived distortion of the test line. As noted in the introduction, this confound is unlikely to be an important factor in the illusion paradigm, and this is borne out by the equivalence in the pattern of errors measured by the two methods.

The results with the modified method seem to indicate that distortions of length do not occur in the aftereffect form of the parallel-lines illusion. Of course, there might be other reasons for this finding. For example, in the absence of the confound, the magnitude of the "true" aftereffect distortion is far too small to be reliably detected by the modified method. Conceivably, also, the assumption that aftereffects of length do not generalize to contours oriented orthogonally to the adapting line may be incorrect. Finally, it is possible that having removed one confound, the modified method has introduced another unknown confound, specific to the modified method, the effect of which is to mask the aftereffect distortion. This seems unlikely, however, inasmuch as a preliminary experiment also failed to measure any aftereffect distortions with a task requiring observers to reproduce the length of a horizontal test line by adjusting the length of a single vertical comparison line. ${ }^{2}$

The length-contrast effects measured by the standard method appear to be consistent with the results of numerous studies, conducted in the figural-aftereffect tradition, 
indicating that the aftereffect forms of many size and area illusions, such as the Müller-Lyer (Kohler \& Wallach, 1944; Pollack, 1964) and Delboeuf (Adam, 1966; Sagara \& Oyama, 1957) illusions, produce contrast. Thus, irrespective of the reasons for the failure to measure length contrast with the modified procedure, contrast effects might appear to be the "best bet" explanation of the aftereffect distortions measured with the standard procedure. Nevertheless, there are reasons for suspending judgment on this issue. First, the figural-aftereffect studies have scrupulously controlled eye movements by instituting the appropriate fixation conditions during both the adapting and the judgment or comparison phases of the experiment, precisely in order to overcome the kinds of problems discussed in the introduction. With the graded-series method, however, the fixation requirement is imposed only during the adapting phase. In view of this fundamental difference, it seems unwise to invoke a contrast explanation of the aftereffect distortions measured with the standard method by appealing to findings obtained under fundamentally different fixation conditions. Second, the consistency argument is not compelling because it assumes that the distortions found in the aftereffect form of the parallellines illusion are similar in kind to those found with other aftereffects of size and area. Yet there are a few instances, such as the Ponzo figure, for which the aftereffect and illusion forms produce a similar pattern of distortions (Kohler \& Wallach, 1944); for both forms the line near the vertex of the angle is judged to be longer than the line near the base of the angle. Moreover, given the ambiguity of the data obtained with the graded-series method, the possibility that assimilatory, rather than contrastive, interactions determines the pattern of aftereffect distortions in the parallel-lines figure cannot be discounted. ${ }^{3}$ These reservations are reinforced by the observation that the parallel-lines aftereffect may not be representative of other aftereffects of length. The adapting stimulus in the parallel-lines configuration-a single line-does not produce an illusion, in the usual sense of the term, whereas for many other aftereffect forms the adapting stimulus is an illusion-producing figure (e.g., the arrows of the Müller-Lyer figure). Viewed in this context, the parallellines aftereffect may represent a class of distortion fundamentally different from those size distortions for which the aftereffect forms require adapting an illusion-producing mechanism.

In summary, the present findings indicate that with the graded-series method the magnitude and the direction of the aftereffect, but not the illusion, of perceived length are dependent on the relative orientations of the test and comparison lines. In particular, these findings suggest that it might be prudent to avoid using this method, at least to measure aftereffect distortions of length. Certainly, until it is established that the method does measure the phenomenon of interest and, specifically, that measurements are not confounded with the measurement device itself, the aftereffect results of studies using the gradedseries method must be interpreted with caution.

\section{REFERENCES}

AD^M, J. (1966). The relationship between visual illusions and figural aftereffects. Australian Journal of Psychology, 18, 130-134.

BRIGELL, M., \& ULARIK, J. (1979). The relational determination of length illusions and length aftereffects. Perception, 8, 187-197.

Coren, S., Girgus, J. S. (1972). A comparison of five measurements of illusion measurement. Behavior Research Methods \& Instrumentation, 4, 240-244.

Coren, S., \& Girgus, J. S. (1978). Seeing is deceiving: The psychology of visual illusions. Hillsdale, NJ: Erlbaum.

Jordan, K., HALEBLIAN, J. (1988). Orientation specificity of length assimilation and contrast. Perception \& Psychophysics, 43, 446-456.

Jordan, K., \& UhlariK, J. (1985). Assimilation and contrast of perceived length depend on temporal factors. Perception \& Psychophysics, 37, 447-454.

KoHLER, W., WaLlaCh, H. (1944). Figural after-effects: An investigation of visual processes. Proceedings of the American Philosophical Society, 88, 269-357.

Pollack, R. H. (1964). Simultaneous and successive presentation of elements of the Mueller-Lyer figure and chronological age. Perceptual \& Motor Skills, 19, 303-310.

PREDEBON, J. (1988). Length distortions in the aftereffect form of the parallel-lines illusion: Contrast or assimilation? Unpublished manuscript.

Sagara, M., \& Oyama, T. (1957). Experimental studies on figural aftereffects in Japan. Psychological Bulletin, 54, 327-338.

\section{NOTES}

1. These findings were confirmed on an independent group of observers.

2. The Macintosh computer was used to present the adapting line, either 2 or $4.5 \mathrm{~cm}$ long, for $60 \mathrm{sec}$, after which it was replaced by the $3-\mathrm{cm}$ long test line together with the adjustable vertical line. By pressing the appropriate key on the computer keyboard, the subjects could either increase or decrease the length of the vertical line. However, because the subjects $(N=9)$ frequently took about $10 \mathrm{sec}$ to complete the adjustment task, it is possible that the null finding reflects the decay of the aftereffect.

3. If it is assumed that (1) assimilatory, not contrastive, interactions characterize the aftereffect distortion, and (2) the aftereffect interacts with the scale illusion, then, on the basis of the illusion data, it is possible to predict both the pattern and the magnitude of the aftereffect errors found in this and in Jordan and Uhlarik's (1985) study (Predebon, 1988).

(Manuscript received November 16, 1988; revision accepted for publication June $13,1989$. 